\title{
Resource Allocation for Femtocell Networks with Imperfect Channel Knowledge
}

\author{
Xudong Kuang, Qinghai Yang, Fenglin Fu \\ State Key Laboratory on ISN, \\ School of Telecommunications Engineering, \\ Xidian University, No.2 Taibainan-lu, \\ Xi' an, 710071, Shaanxi,China. \\ Email:qhyang@xidian.edu.cn
}

\author{
Kyung Sup Kwak \\ Graduate School of Information Technology \\ and Telecommunications, Inha University, \\ \#253 Yonghyun-dong, Nam-gu, \\ Incheon, 402-751, Korea. \\ Email: kskwak@inha.ac.kr
}

\begin{abstract}
We propose an efficient resource allocation scheme for OFDMA based femtocells networks under imperfect channel knowledge. Several femtocell users share the same spectrum but no interference exists between each other. The transmit power of femtocell users is minimized under the users' QoS constraints. Based on the interference channel model of femtocell users, an accurate interference estimation approach is developed. Simulation results show that the proposed algorithm reduces the interference of macro users and increases the spectral efficiency.
\end{abstract}

Index Terms-femtocells, spectral efficiency, resource allocation, QoS, imperfect channel knowledge

\section{INTRODUCTION}

Recently, the growth of the indoor communications overloads the conventional macrocellular systems. Surveys present that fifty percent of phone calls and seventy percent of data services will be happened indoors. Since extending coverage and providing quality of service (QoS) for indoor users require expensive costs to the macrocells, network operators need more efficient solutions for the indoor services [1].

A femto-base station is a small cellular base station (BS) incorporating typical macro-BS functionalities, with less sophisticated deployment which enables an easy use by the subscribers [2]. It has a low transmit power and communicates with the cellular provider's core network via a wired broadband connection such as residential DSL (Digital Subscriber Line) or cable modem [3].

However, introducing femto-BSs in a cellular network presents a major challenge. Actually, despite their low transmit power, these femtocells are often regarded as hostile interferers by macrocells [4]. In fact, the expected femtocells' deployment in the cellular network can lead to significant interference which affects harmfully the macrocells users' QoS. This motivates us to investigate an efficient radio resource allocation scheme for femtocells to attain a successful macro-femto-BSs' co-deployment.

\footnotetext{
*This research was supported by the RCUK for the UK-China Science Bridges Project: R\&D on (B) $4 \mathrm{G}$ wireless mobile communications, the MKE, Korea, under the ITRC program supervised by the NIPA (NIPA-2011-C10901121-0001) and the Fundamental Research Funds for the Central Universities (JY10000901002, JY10000901020), NSF China (60832001, 61001127), the 111 Project (B08038), China.
}

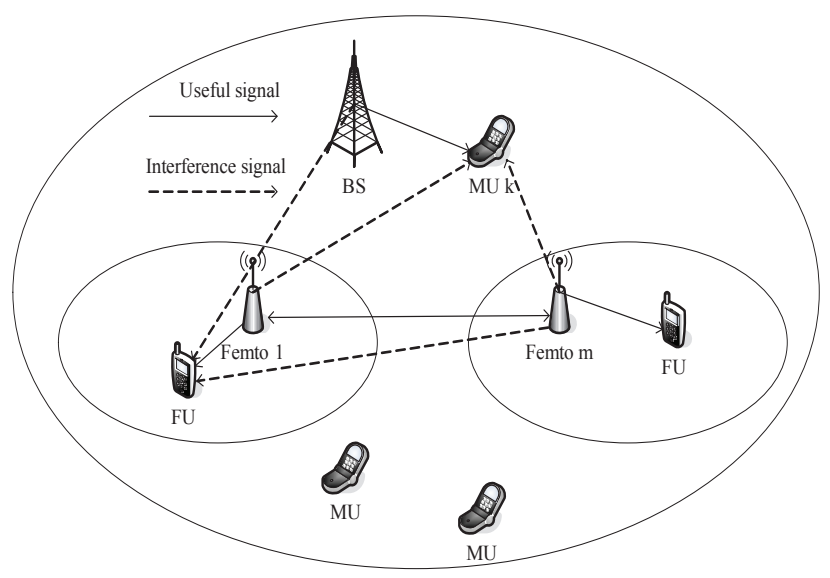

Fig. 1. Interference model in the femtocell networks

In this paper, the subchannel selection is investigated for the OFDMA-based femtocell systems to reduce interference and to increases the spectral efficiency. In the spectrum sharing scheme, several femtocell users share the same frequency without interference to each other. Meanwhile, we minimize the transmit power of femtocell users under users' QoS constraints, and estimate the interference level of a femtocell user based on imperfect channel knowledge.

\section{SYSTEM MODEL}

\section{A. Femtocell Network Model}

Fig. 1 shows a two layer network which consists of macro users (MU) and femtocell users (FU). Suppose that there are $K$ MUs and $M$ FUs, which are randomly located inside the coverage area of the macrocell. A femtocell has only one FU. We assume that the femtocell and the BS exchange information interactively and femtocells exchange information between each other. Due to the delay or the estimation error, the observed information after exchange may not be perfect. We assume that the total bandwidth is divided into $N$ subcarriers with two of them being grouped into one subchannel. Both macrocell and femtocell operate in the same frequency band and have the same amount of available subcarriers. 


\section{B. Interference Avoidance Model Between Femtocell and MU}

The spectrum sharing by FU should guarantee the normal transmission of MU. The total transmit power of FU in each channel is no more than the interference power threshold $P_{\text {safe }}$, which is a MU can endure. Defining the maximum transmit power of a FU as $P_{\max }$, we can write

$$
\sum_{m=1}^{M} P_{m}^{n} G_{m, k}^{n} \leq P_{\text {safe }},
$$

where $G_{m, k}^{n}$ is channel gain between femtocell $m$ and MU $k$ over the subchannel $n$. The transmit power $P_{m}^{n}$ of FU $m$ on subchannel $n$ should satisfy

$$
P_{\min } \leq P_{m}^{n} \leq P_{\max }
$$

\section{Problem Formulation}

The downlink signal to interference plus noise ratio (SINR) from femtocell $m$ to FU $m$ over the subchannel $n$ is given by

$$
S I N R_{m, n}=\frac{P_{m}^{n} H_{f, m}^{n}}{N_{0} W^{n}+\sum_{k \in K} P_{k}^{n} G_{k, m}^{n} * H_{k, m}^{n}+\sum_{m^{\prime} \in M^{\prime}} P_{m^{\prime}}^{n} G_{m^{\prime}, m}^{n}},
$$

where $H_{f, m}^{n}$ is the channel gain between FU $m$ and femtocell $m$ on subchannel $n, N_{0}$ is the power spectral density of the white Gaussian noise, $W^{n}$ is the bandwidth of subchannel n. $G_{k, m}^{n}$ is the path loss between BS and FUm, $H_{k, m}^{n}$ is the frequency selective fading on the channel $n$ between BS and FU $m . P_{m^{\prime}}^{n} G_{m^{\prime}, m}^{n}$ is the interference from other femtocells. Because the family station is installed indoors, we only consider path loss and disregard frequency selective fading between femtocells. The co-channel interference (CCI) on subchannel $n$ of FU $m$ in the coverage of femtocell $m$ is defined as

$$
I_{m}^{n}=\sum_{k \in K} P_{k}^{n} G_{k, m}^{n} * H_{k, m}^{n}+\sum_{m^{\prime} \in M^{\prime}} P_{m^{\prime}}^{n} G_{m^{\prime}, m}^{n},
$$

where $G=10^{-P L / 10}$.

\section{A. Imperfect Channel Information}

We assume a femtocell allocates the resources and makes decisions for FU based on CSI feedback from BS. However, due to the feedback delay, the femtocell does not have the perfect CSI but usually outdated. We consider a stationary ergodic Gauss-Markov block fading process. The channel variation on subcarrier $n$ between instants $t$ and $(t-\tau)$ is modeled as

$$
H_{k, m}^{n}(t)=\rho_{k, m} H_{k, m}^{n}(t-\tau)+e_{k, m}^{n}(t),
$$

where the subscript $k, m$ denotes the channel coefficient of the subcarrier $n$ from node $k$ to node $m, \tau$ is the feedback delay, $\rho_{k, m}$ is the time-auto correlation,

$$
\rho_{k m}=\mathcal{E}\left[H_{k, m}^{n}(t) * H_{k, m}^{n}(t-\tau)^{*}\right] .
$$

The channel error $e_{k, m}^{n}(t)$ is defined as

$$
e_{k, m}^{n}(t)=\sqrt{1-\rho_{k, m}^{2}} \mu_{k, m}^{n}(t),
$$

where $\mu_{k, m}^{n}(t)$ is a complex Gaussian random variable with zero mean and unit variance.

\section{B. Problem Description}

The data rate achieved by the FU $m$ over subchannel $n$ is represented

$$
r_{m}^{n}=W^{n} \ln \left(1+S I N R_{m, n}\right) .
$$

Without loss of generality, we assume the FU $m$ has the target data rate $\mathrm{R}_{m}$. In the downlink, the CCI from femtocells to macrocell users has to be minimized. In order to reduce the $\mathrm{CCI}$, the femtocell BS requires to transmit power as small as possible. Therefore, our objective is to minimize the total transmission power of the femtocells. The downlink resource allocation problem of the femtocell BS $m$ is formulated as.

$$
\mathcal{P}=\min \sum_{m \in M} \sum_{n=1}^{N} a_{m, n} P_{m}^{n},
$$

subject to

$$
\begin{gathered}
\sum_{n=1}^{N} a_{m, n} r_{m}^{n}=\mathrm{R}_{m}, \forall m, \\
0 \leq \sum_{i=1}^{M} P_{m}^{n} G_{m, k}^{n} \leq P_{\text {safe }}, \forall n \\
P_{\min } \leq P_{m}^{n} \leq P_{\max }, \forall n \\
a_{m, n} \in(0,1) .
\end{gathered}
$$

Constraint (10) guarantees the service rate requirements of all users; The total transmit power of a FU in each channel is no more than the interference power threshold $P_{\text {safe }}$, as noted by constraint (11); $a_{m, n}$ in constraint (13) is the allocation indicator.

Obviously, the above problem is an NP-hard combinatorial optimization problem with non-linear constraints. Finding an optimal solution directly is computationally prohibitive. As the number of subcarriers and FUs increases, the number of combinations for $a_{m, n}$ increases exponentially. Therefore, we adopt a tow-step approach to reduce the computational complexity. That is, we decouple the problem into the subcarrier allocation and the power allocation problems. In the subcarrier allocation step, we proposed an efficient spectrum allocation scheme and obtain the allocation matrix. In the power allocation step, the power is assigned to each selected subchannel to minimize the total power consumption and to satisfy user's QoS jointly.

\section{Subchannel Allocation}

\section{A. Subchannel Allocation Algorithm}

Free matrix $L=\left\{l_{m, n} \mid l_{m, n} \in(0,1)\right\}_{M \times N}$ describes whether channel $n$ is available to FU $m$, where $l_{m, n}=1$ means that channel $n$ is available to FU $m$. Interference matrix $C=\left\{c_{m, k, n} \mid c_{m, k, n} \in(0,1)\right\}_{M * M * N}$ describes the interference between FUs. If $c_{m, k, n}=1, \mathrm{FU} m$ and FU $k$ cannot use channel $n$ simultaneously. We set $c_{m, k, n}=1-l_{m, n}$. The interference between different users dominated by their position relations and doesn't change during the allocation 
interval. The interference matrix is determined by exchanging location information of femtocells. Allocation matrix $A=$ $\left\{a_{m, n} \mid a_{m, n} \in(0,1)\right\}_{M \times N}$ shows channel allocation situation. $a_{m, n}=1$ means channel $n$ is allocated to FU $m$. Allocation matrix must satisfy some constraints. A channel available to FU will not be allocated to existing interference FUs. Transmission requirements vector $R=\left\{r_{i} \mid r_{i} \in(0,1)\right\}_{M \times 1}$ shows transmission requirements of femtocell. $r_{i}=1$ means femtocell $i$ has data for transmission.

To share the spectrum, when a FU $m$ has transmission requirement, the FU $m$ firstly chooses an optimal subcarrier $n$ * by Eq. (15). Then femtocell determines whether interference exists between the FU $m$ and other FUs which are using subcarrier $n^{*}$ according to the interference matrix $C$. If there is no interference, the subcarrier $n^{*}$ is allocated to FU $m$. Otherwise, the subcarrier $n^{*}$ is not allocated to FU $m$. And then the subcarrier $n^{*}$ is deleted from $L$. Let repeat the above steps until meeting the rate requirement of FU $m$. The algorithm is performed until all available subcarriers are allocated.

The carrier to interference ratio (CIR) of each channel $1 \leq$ $n \leq N$ can be calculated for FU $m(m=1,2, \cdots, M)$ :

$$
g_{m, n}=\frac{H_{f, m}^{n}}{N_{0} W^{n}+\sum_{k \in K} P_{k}^{n} G_{k, m}^{n} H_{k, m}^{n}+\sum_{m^{\prime} \in M^{\prime}} P_{m^{\prime}}^{n} G_{m^{\prime}, m}^{n}} .
$$

FU $m$ selects the optimal subchannel $n^{*}$ :

$$
n^{*}=\arg \max _{n} g_{m, n} .
$$

The detailed algorithm is summarized in Algorithm 1. Note that we drop the subscript $m$. Each femto-BS calculates CIR of each channel for FU. According to Eq.(15), each femtoBS scans the free matrix $L$, then selects the optimal subcarrier that presents the maximum CIR. We must guarantee that the selected subcarrier cannot be used by other interfering FUs.

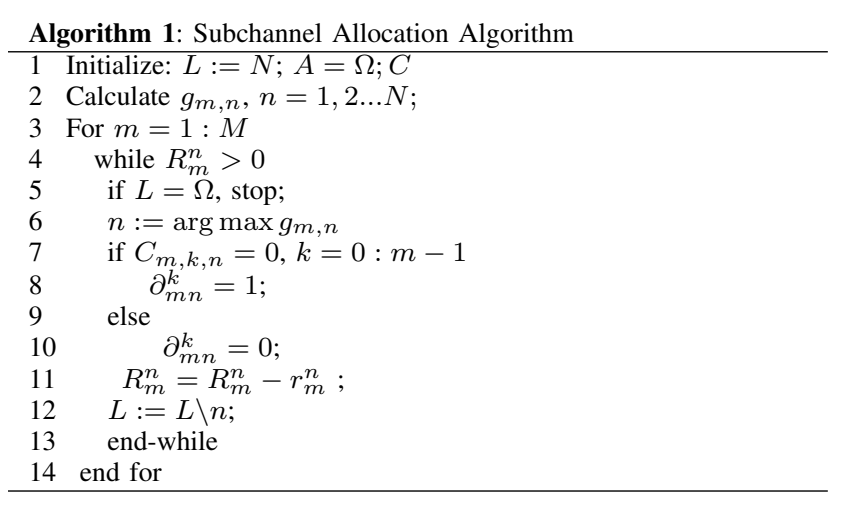

\section{Total Transmit Power Minimization}

\section{A. Power Allocation}

In the power allocation step, the femto-BS minimizes the transmit power of subchannels to suppress the CCI. Since allocation matrix $A$ is determined in the channel allocation step, the problem (4) becomes a simple minimization problem for $P_{m}^{n}$ with inequality constraints:

subject to

$$
\min \sum_{n=1}^{N} a_{m, n} P_{m}^{n}
$$

$$
\begin{aligned}
& \mathrm{R}_{m}-\sum_{n=1}^{N} a_{m, n} r_{m}^{n}=0, \forall m, \\
& P_{\text {safe }}-\sum_{i=1}^{M} P_{m}^{n} G_{m, k}^{n} \geq 0, \forall n \\
& P_{\min } \leq P_{m}^{n} \leq P_{\max }, \forall n .
\end{aligned}
$$

The femto-BS $m$ solves this problem for each user $m$ independently. The Karush-Kuhn-Tucker (KKT) conditions of this problem are presented as:

$$
\begin{gathered}
a_{m}^{n}\left\{1-\frac{\lambda W^{n} H_{f, m}^{n}}{P_{m}^{n} H_{f, m}^{n}+I_{m}^{n}+N_{0} W^{n}}+u^{n} G_{m, k}^{n}\right\}=0 \\
u^{n}\left(P_{\text {safe }}-\sum_{i=1}^{M} P_{m}^{n} G_{m, k}^{n}\right)=0, \forall n \\
u^{n} \geq 0, \forall n
\end{gathered}
$$

where $\lambda$ is the Lagrange multiplier and $u^{n}$ is the KKT multipliers. Since the problem specifies the user $m$, we only consider subchannel $n$ with $a_{m, n}=1$. Hence, the transmit power of subcarrier $n$ derived from (17) is

$$
P_{m}^{n}=\frac{\lambda W^{n}}{1+u^{n} G_{m, k}^{n}}-\frac{I_{m}^{n}+N_{0} W^{n}}{H_{f, m}^{n}} .
$$

So, the optimal solution is obtained as:

$$
P_{m}^{*}=\left\{\begin{array}{l}
P_{\min }, \\
\frac{\lambda W^{n}}{1+u^{n} G_{m, k}^{n}}-\frac{I_{m}^{n}+N_{0} W^{n}}{H_{f, m}^{n}}, P_{\min } \leq P_{\min } \leq P_{m}^{n} \leq P_{\max } \\
P_{\max }, \quad P_{m}^{n}>P_{\max } .
\end{array}\right.
$$

\section{B. Convergence Analysis}

For $P_{m}=\lambda W^{n} /\left(1+u^{n} G_{m, k}^{n}\right)-\left(I_{m}^{n}+N_{0} W^{n}\right) / H_{f, m}^{n}$ in (22), the iterative formula $p_{m}$ is:

$$
\begin{aligned}
& P_{m}(n)=\frac{\lambda W^{n}}{1+u^{n} G_{m, k}^{n}}- \\
& \frac{\sum_{k \in K} P_{k}^{n} G_{k, m^{n}}^{n} * H_{k, m}^{n}+\sum_{m^{\prime} \in M^{\prime}} G_{m^{\prime}, m}^{n} P_{m^{\prime}}(n-1)+N_{0} W^{n}}{H_{f, m}^{n}} .
\end{aligned}
$$

To verify the convergence of (22), we prove that the difference of two iteration value is a convergent series.

According to (22), we can obtain the gap value between the iteration $n+1$ and the iteration $n$ as,

$$
P_{m}(n+1)-P_{m}(n)=\frac{\sum_{m^{\prime} \in M^{\prime}} G_{m^{\prime}, m}^{n}\left(P_{m^{\prime}}(n)-P_{m^{\prime}}(n-1)\right)}{H_{f, m}^{n}} .
$$




$$
\begin{aligned}
& \forall j \in N, \exists \Delta,\left|P_{m}(n+1)-P_{m}(n)\right| \leq \Delta, \text { so } \\
& \frac{\left|P_{i}(n+1)-P_{i}(n)\right|}{\Delta} \leq \frac{H_{f, m}^{n}}{\sum_{m^{\prime} \in M^{\prime}} G_{m^{\prime}, m}^{n}} .
\end{aligned}
$$

The convergence condition of (22) is given by

$$
\forall m, m^{\prime} \in M, \frac{H_{f, m}^{n}}{\sum_{m^{\prime} \in M^{\prime}} G_{m^{\prime}, m}^{n}} \geq 1 .
$$

Equation (25) is equivalent to each FU's CINR requirement. The above condition can be managed by access control. By above proof, the power solution not only exists but also converges.

\section{AcCess Control Scheme}

When there are massive femtocells in the system, the interference to MU may be quite strong, so we have to control of the new femtocell access. We propose a novel power allocation algorithm, which guarantees that the interference level observed at each $\mathrm{MU}$ is under the value $P_{\text {safe }}$. Thus, it guarantees that the total transmit power of $\mathrm{FU}$ in each subchannel does not surpass the interference power threshold. That is, FU access does not affect the normal communication of MU. Hence, we develop an access control algorithm, which can admit or reject femtocell to access. Each MU of the LTE system will calculate its received signal strength indication (RSSI) and carrier to interference noise ratio (CINR) and return the result of the measurement to its BS. BS may use the feedback to estimate the interference level at each MU. When the interference intensity observed at MU does not meet Eq.(1)BS reports it to the femtocell gateway. With the vector of transmit requirements $R=\left\{r_{i} \mid r_{i} \in(0,1)\right\}_{M \times 1}$, femtocell gateway does not allow the new femtocell to access. For example, in accordance with the sequencing of femtocell user, when (1) is not satisfied, the femtocell gateway will reject the new femtocell user to access.

\section{Simulation Results}

We evaluate the performance of the proposed resource allocation algorithm via simulations. We consider an OFDMA cellular system. The simulation parameters are given in TABLE I. The channel between BS and FU is represented as a combination of path loss fading and frequency selective lognormal fading. We only consider path loss and do not consider frequency selective fading between femtocells. Femtocell BSs are uniformly distributed in the macrocells. We consider that minimum interference distance of $\mathrm{FU}$ is $0.7 \mathrm{Km}$. When the distance between two FUs is more than $0.7 \mathrm{Km}$, we assume they might share the same sabcarrier. We assume that there are only one user in a femtocell and the service rate requirement of a femtocell user is $1 \mathrm{Mbps}$ equally. We assume that macrousers are always outdoor and femtousers are always indoor.

Fig. 1 shows the MU interference from femtocells. In contrast to the random selection scheme, the proposed channel selection schemes decreases the MU interference from femtocells. Meanwhile, the interference intensity of the proposed shared-spectrum allocation scheme is the same as the exclusive-spectrum scheme. We also observe that the maximum interference power of $\mathrm{MU}$ is less than $22 \mathrm{dBm}$ by the proposed channel selection schemes. So, the proposed interference avoidance scheme can decrease the MU interference from femtocells.

TABLE I

SIMULATION PARAMETERS

\begin{tabular}{|l|r|}
\hline Parameters & Values \\
\hline Macrocell Radius & $1 \mathrm{Km}$ \\
\hline Minimum interference distance of FU & $0.7 \mathrm{Km}$ \\
\hline Subcarriers Number & 1000 \\
\hline Subcarrier Bandwidth & $15 \mathrm{KHZ}$ \\
\hline Transmit power of BS & $46 \mathrm{dBm}$ \\
\hline Maximum transmit power of femtocell & $10 \mathrm{dBm} ;$ \\
\hline Minimum transmit power of femtocell & $5 \mathrm{dBm}$ \\
\hline Minimum rate of FU & $1 \mathrm{Mbps}$ \\
\hline Number of femtocells & 200 \\
\hline Number of MUs & 50 \\
\hline Maximum interference power of MU & $25 \mathrm{dBm}$ \\
\hline Path loss from macro BS to femto users & $28+35 \log 10(\mathrm{~d}(\mathrm{~m}))+\mathrm{WP}$ \\
\hline Path loss from femto BS to macro & $7+56 \log 10(\mathrm{~d}(\mathrm{~m}))+\mathrm{WP}$ \\
\hline Path loss from femto BS to own users & $37+35 \log 10(\mathrm{~d}(\mathrm{~m}))$ \\
\hline
\end{tabular}

Fig. 2 depicts the throughput of macrocell against the number of femtocells in the proposed shared-spectrum allocation scheme and exclusive-spectrum scheme. As the number of femtocells increases, the throughput of macrocell decreases due to the increasing interference from the femtocells. Compared with the exclusive-spectrum scheme, the proposed scheme effectively decreases interference and improves the capacity of macrocell. When the femtocells increased to 160 , the capacity of macrocell decreased below to $70 \mathrm{Mbit} / \mathrm{s}$. So, we should not allow new femtocell to access according to access control scheme.

Fig.3 illustrates the spectral efficiency for various numbers of femtocells in different spectrum allocation scheme. When the number of femtocells is small, spectral efficiency of the proposed shared-spectrum allocation scheme is the same as the exclusive-spectrum scheme and is higher than the random selection scheme. Compared with other selection schemes, the proposed scheme has highter spectral efficiency since the femtocell can use more subchannels to sent data with required interference intensity. In the example, the maximum spectral efficiency of the random selection scheme is less than 0.3. However, the proposed scheme can increase spectral efficiency to 0.65 .

\section{CONCLUSIONS}

In this paper, we proposed a shared-spectrum allocation scheme to guarantee FU QoS and to reduce the interference to macro users in OFDMA-based femtocell systems. In the femtocells, the femtocell-to-macrocell interference significantly degrades the capacity of macrocell. The proposed interference avoidance scheme can decrease the MU interference from femtocells. Meanwhile, we developed an efficient resource allocation algorithm to increase spectral efficiency. The performance of the proposed resource management algorithm is demonstrated by simulations. 


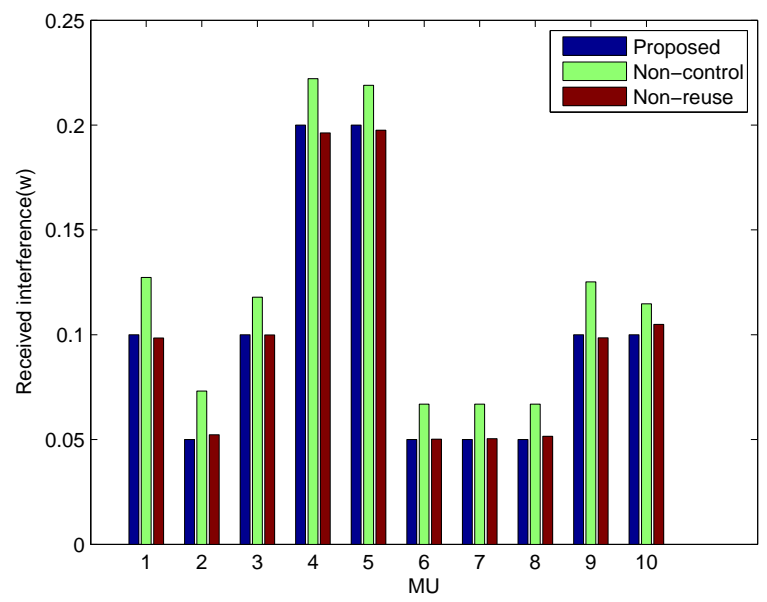

Fig. 2. Received interference of MU

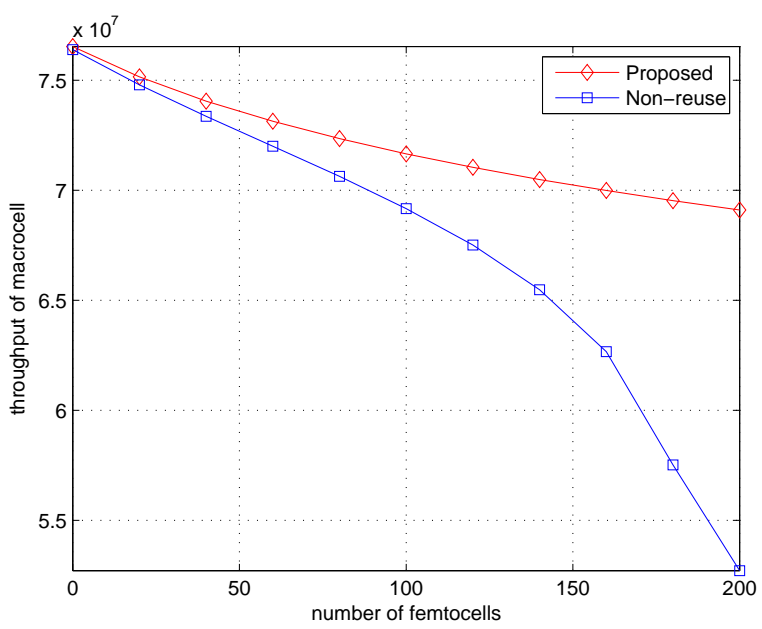

Fig. 3. Throughput of Macrocell

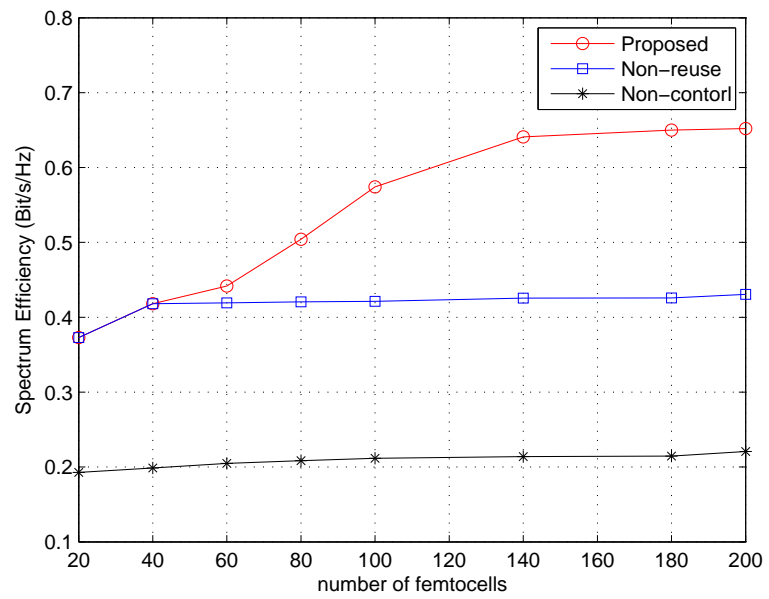

Fig. 4. Spectral Efficiency

\section{REFERENCES}

[1] L.P.David, A.Valcarce and G.de laRoche, "OFDMA femtocells: a roadmap on interference avoidance," IEEE Commun. Mag, vol.47, no. 9, pp. 41-48, Sep. 2009.

[2] S.Ortiz, "The wireless industry begins to embrace femtocells," IEEE Computer Society, vol.41, IsFU 7 , pp. 14 - 17 July 2008.

[3] V. Chandrasekhar, J. Andrew, and A. Gatherer, 'Femtocell networks: A survey," IEEE Commu. Magazine ,vol.46, IsFU 9, pp.59 - 67 Sep. 2008.

[4] G.E.M. Zhioua, P. Godlewski, and S. Hamouda, "Quietest channel selection for femtocells in OFDMA Networks," Proc. of MobiWac'10 , Oct. 2010.

[5] H. Claussen, "Performance of Macro- and Co-Channel femtocells in a Hierarchical cell structure",Personal, Indoor and Mobile Radio Communications, Proc. of IEEEPIMRC 2007,pp.1-5,Sept. 2007.

[6] S. Yeh, S. Talwar and S. Lee, "WiMAX femtocells: A perspective on network architecture, capacity, and coverage," IEEE Commu. Magazine, ,Vol. 46, IsFU 10, pp.58-65, Oct. 2008.

[7] M. D. Katz, and F. H.P. Fitzek, "WiMAX evolution: emerging technologies and applications," Wiley publication ,2009.

[8] IEEE Standard for local and metropolitan area networks, Part 16: Air Interface for Fixed and Mobile Broadband Wireless Access Systems, Amendment 2: Physical and Medium Access Control Layers for Combined Fixed and Mobile Operation in Licensed Bands and Corrigendum $1,2005$.

[9] D. Lopez-Perez, G. De La Roche, and A. Valcarce, "Interference avoidance and dynamic frequency planning for WiMAX femtocells networks," 11th IEEE Singapore International Conference, pp.1579-1584, Nov. 2008.

[10] G.E.M. Zhioua, P. Godlewski, and S. Hamouda, "Quietes channel selection for femtoCells in OFDMA networks," Proc. of MobiWac'10, Oct. 2010. 